\title{
A rule-based framework for heterogeneous subsystems management in smart home environment
}

\begin{abstract}
Recent advancements in computing and communication technologies have increased the growth of heterogeneous subsystems in smart home environment. However, many of these heterogeneous systems are standalone and do not adapt towards joint execution of tasks. Hence, it is rather difficult to perform interoperation especially to realize desired services preferred by home dwellers. In this paper, we propose a new rule-based framework for heterogeneous systems management as well as coordinating them by means of federated manner in smart home environment. The proposed framework is based on event-conditionaction (ECA) rule mechanism with SOAP technology that provides interoperability among those systems. We have implemented the framework with several subsystems to demonstrate their effectiveness for interoperation using ECA rule mechanism. The performance of the framework was tested in LAN environment and proves to be reliable in smart home setting.
\end{abstract}

Keyword: ECA; Smart home; SOAP 